Supporting information for

\title{
Enhanced Dibenzothiophene Capture with Multmetal-Organic Frameworks in Presence of Benzene and Octane
}

\author{
Yuan Gao,${ }^{\dagger}$ Jiafei Wu,${ }^{\dagger}$ Xiaoqin Xiong, ${ }^{\dagger} \mathrm{Na}$ Yan $,{ }^{\dagger} \mathrm{Na} \mathrm{Ma},{ }^{\dagger}$ and Wei Dai ${ }^{*}, \dagger$ \\ ${ }^{\dagger}$ Key Laboratory of the Ministry of Education for Advanced Catalysis Materials, College of \\ Chemistry and Life Science, Zhejiang Normal University, Jinhua 321004, People's Republic of \\ China \\ †College of Geography and Environmental Sciences, Zhejiang Normal University, Jinhua, \\ Zhejiang Province 321004, China
}

Corresponding author: Wei Dai

E-mail address: daiwei@zjnu.edu.cn 
Table S1. Textural structure properties of $\mathrm{Cu}-\mathrm{BTC}$ and MMOFs.

\begin{tabular}{cccc}
\hline Samples & $\mathrm{S}_{\text {BET }}\left(\mathrm{m}^{2} / \mathrm{g}\right)$ & $\mathrm{V}_{\text {total }}(\mathrm{mL} / \mathrm{g})$ & Average pore diameter $(\mathrm{nm})$ \\
\hline $\mathrm{Cu}-\mathrm{BTC}$ & 1243 & 0.58 & 2.15 \\
$(2.0) \mathrm{Ag} / \mathrm{Cu}-\mathrm{BTC}$ & 1571 & 0.63 & 1.62 \\
$(3.0) \mathrm{Ag} / \mathrm{Cu}-\mathrm{BTC}$ & 1549 & 0.61 & 1.72 \\
$(4.0) \mathrm{Ag} / \mathrm{Cu}-\mathrm{BTC}$ & 1541 & 0.54 & 1.70 \\
$(5.0) \mathrm{Ag} / \mathrm{Cu}-\mathrm{BTC}$ & 1489 & 0.45 & 1.64 \\
$(4.0) \mathrm{Ag} / \mathrm{Zn} / \mathrm{Cu}-\mathrm{BTC}$ & 1055 & 0.41 & 1.93 \\
$(4.0) \mathrm{Ag} / \mathrm{Zn} / \mathrm{Ni} / \mathrm{Cu}-\mathrm{BTC}$ & 633 & 0.36 & 3.16 \\
\hline
\end{tabular}

Table S2. Comparisons of DBT S-capacity adsorbed onto different adsorbents.

\begin{tabular}{cccc}
\hline Adsorbents & Solvents & $q_{\max }(\mathrm{mg} \mathrm{S} / \mathrm{g})$ & References \\
& ALF & 85 & \\
$\mathrm{Ag} / \mathrm{Zn} / \mathrm{Ni} / \mathrm{Cu}-\mathrm{BTC}$ & ARF & 103 & this work \\
& MIF & 95 & \\
Carbon-doped porous boron nitride & ALF & 35 & $(1)$ \\
UMCM-150 & ALF & 83 & $(2)$ \\
MIL-53(Cr) & ALF & 86 & $(3)$ \\
MIL-100(Fe) & ALF & 39 & $(4)$ \\
$\mathrm{AgO}_{3} \mathrm{~S}-\mathrm{MIL}-101(\mathrm{Cr})$ & ALF & 31 & $(4)$ \\
Co-Y zeolite & ALF & 29 & $(5)$ \\
Ce/Ni-Y zeolite & ALF & 22 & $(6)$ \\
Activated $\mathrm{Al}_{2} \mathrm{O}_{3}$ & n-hexane & 21 & $(7)$ \\
CMK-3 & n-hexane & 11 & $(8)$ \\
IRMOF-3@ $\gamma-\mathrm{Al}_{2} \mathrm{O}_{3}$ & ALF & 55 & $(9)$ \\
IRMOF-3 & ALF & 45 & $(9)$ \\
MOF-5@ $\gamma-\mathrm{Al}_{2} \mathrm{O}_{3}$ & ALF & 31 & $(9)$ \\
MOF-5 & ALF & 24 & $(9)$ \\
\hline
\end{tabular}

*ALF=aliphatic fuel (Isooctane), ARF=aromatic fuel (benzene), MIF=mixed fuel (20 wt $\%$ benzene and $80 \mathrm{wt} \%$ isooctane).

\section{References:}

(1) Xiong, J.; Zhu, W.; Li, H.; Yang, L.; Chao, Y.; Wu, P.; Xun, S.; Jiang, W.; Zhang, M.; Li, H.Carbon-doped porous boron nitride: metal-free adsorbents for sulfur removal from fuels. $J$. Mater. Chem. A 2015, 3, 12738- 12747

(2) Cychosz, K. A.; Wong-Foy, A. G.; Matzger, A. J. Liquid Phase Adsorption by Microporous Coordination Polymers: Removal of Organosulfur Compounds. J. Am. Chem. Soc. 2008, 130,6938- 6939 
(3) Han, L.; Zhang, J.; Mao, Y.; Zhou, W.; Xu, W.; Sun, Y. Facile and green synthesis of MIL-53(Cr) and its excellent adsorptive desulfurization performance. Ind. Eng. Chem. Res. 2019, $58,34,15489-15496$.

(4) Khan, N. A.; Jhung, S. H. Adsorptive removal and separation of chemicals with metal-organic frameworks: contribution of $\pi$-complexation. J. Hazard. Mater. 2017, 325, 198-213.

(5) Wang, J.; Xu, F.; Xie, W. J.; Mei, Z. J.; Zhang, Q. Z.; Cai, J.; Cai, W. M. The enhanced adsorption of dibenzothiophene onto cerium/nickel-exchanged zeolite Y. J. Hazard. Mater. 2009, $163,538-543$.

(6) Srivastav, A.; Srivastava, V. C. Adsorptive desulfurization by activated alumina. J. Hazard. Mater. 2009, 170, 1133-1140.

(7) Nejad, N. F.; Shams, E.; Amini, M. K.; Bennett, J. C. Ordered mesoporous carbon CMK-5 as a potential sorbent for fuel desulfurization: application to the removal of dibenzothiophene and comparison with CMK-3. Microporous Mesoporous Mater. 2013, 168, 239-246.

(8) Shi, Y.; Zhang, X.; Wang L.; Liu, G. MOF-derived porous carbon for adsorptive desulfurization. AIChE J. 2014, 60, 2747-2751.

(9) Zuhra, Z.; Mu, C.; Tang, F.; Zhou, Y.; Zhang, L.; Zhao Z.; Qin, L. Enhanced adsorptive desulfurization by iso-structural amino bearing IRMOF-3 and IRMOF-3@ $\mathrm{Al}_{2} \mathrm{O}_{3}$ versus MOF-5 and MOF-5@ $\mathrm{Al}_{2} \mathrm{O}_{3}$ revealing the predominant role of hydrogen bonding. Dalton Trans., 2019, 48, 14792-14800. 
Table S3. Constants and correlation coefficients of different adsorption models in ALF.

\begin{tabular}{|c|c|c|c|c|c|c|c|c|c|c|c|c|}
\hline \multirow[b]{2}{*}{ Samples } & \multicolumn{3}{|c|}{ Langmuir } & \multicolumn{3}{|c|}{ Freundlich } & \multicolumn{3}{|c|}{ Temkin } & \multicolumn{3}{|c|}{ D-R } \\
\hline & $\begin{array}{c}q_{L} \\
(\mathrm{mg} / \mathrm{g})\end{array}$ & $\begin{array}{c}K_{L} \\
(\mathrm{~L} / \mathrm{mg})\end{array}$ & $R^{2}$ & $\begin{array}{c}K_{f} \\
(\mathrm{~L} / \mathrm{g})\end{array}$ & $n$ & $R^{2}$ & $\begin{array}{c}b_{T} \\
(\mathrm{~kJ} / \mathrm{mol})\end{array}$ & $\begin{array}{c}K_{T} \\
(\mathrm{~L} / \mathrm{g})\end{array}$ & $R^{2}$ & $\begin{array}{c}q_{s} \\
(\mathrm{mg} / \mathrm{g})\end{array}$ & $\begin{array}{c}K_{D} \\
\left(\mathrm{~mol}^{2} / \mathrm{kJ}^{2}\right)\end{array}$ & $R^{2}$ \\
\hline $\mathrm{Cu}-\mathrm{BTC}$ & 52.74 & 0.0128 & 0.99 & 11.52 & 4.70 & 0.73 & 295.27 & 0.376 & 0.75 & 49.05 & 0.0016 & 0.90 \\
\hline (2.0)Ag/Cu-BTC & 59.21 & 0.0114 & 0.99 & 11.04 & 4.24 & 0.76 & 245.76 & 0.250 & 0.80 & 54.40 & 0.0017 & 0.96 \\
\hline (3.0)Ag/Cu-BTC & 61.12 & 0.0157 & 0.99 & 14.19 & 4.82 & 0.80 & 262.76 & 0.516 & 0.83 & 56.24 & 0.0008 & 0.91 \\
\hline (4.0)Ag/Cu-BTC & 68.21 & 0.0187 & 0.99 & 17.38 & 5.07 & 0.85 & 249.86 & 0.835 & 0.86 & 61.58 & 0.0003 & 0.81 \\
\hline (5.0)Ag/Cu-BTC & 63.82 & 0.0168 & 0.99 & 15.09 & 4.85 & 0.81 & 253.85 & 0.571 & 0.83 & 58.52 & 0.0006 & 0.88 \\
\hline (4.0)Ag/Zn/Cu-BTC & 70.67 & 0.0486 & 0.99 & 26.06 & 6.66 & 0.85 & 307.93 & 7.863 & 0.88 & 66.26 & 0.0005 & 0.88 \\
\hline (4.0)Ag/Zn/Ni/Cu-BTC & 84.60 & 0.0456 & 0.99 & 25.66 & 5.39 & 0.78 & 219.24 & 2.600 & 0.83 & 77.86 & 0.0001 & 0.87 \\
\hline
\end{tabular}

Table S4. Constants and correlation coefficients of different adsorption models in ARF.

\begin{tabular}{|c|c|c|c|c|c|c|c|c|c|c|c|c|}
\hline \multirow[b]{2}{*}{ Samples } & \multicolumn{3}{|c|}{ Langmuir } & \multicolumn{3}{|c|}{ Freundlich } & \multicolumn{3}{|c|}{ Temkin } & \multicolumn{3}{|c|}{ D-R } \\
\hline & $\begin{array}{c}q_{L} \\
(\mathrm{mg} / \mathrm{g})\end{array}$ & $\begin{array}{c}K_{L} \\
(\mathrm{~L} / \mathrm{mg})\end{array}$ & $R^{2}$ & $\begin{array}{c}K_{f} \\
(\mathrm{~L} / \mathrm{g})\end{array}$ & $n$ & $R^{2}$ & $\begin{array}{c}b_{T} \\
(\mathrm{~kJ} / \mathrm{mol})\end{array}$ & $\begin{array}{c}K_{T} \\
(\mathrm{~L} / \mathrm{g})\end{array}$ & $R^{2}$ & $\begin{array}{c}q_{s} \\
(\mathrm{mg} / \mathrm{g})\end{array}$ & $\begin{array}{c}K_{D} \\
\left(\mathrm{~mol}^{2} / \mathrm{kJ}^{2}\right)\end{array}$ & $R^{2}$ \\
\hline $\mathrm{Cu}-\mathrm{BTC}$ & 64.39 & 0.0100 & 0.99 & 12.86 & 4.55 & 0.83 & 234.20 & 0.264 & 0.85 & 58.00 & 0.0018 & 0.88 \\
\hline (2.0)Ag/Cu-BTC & 71.43 & 0.0097 & 0.99 & 14.01 & 4.76 & 0.87 & 217.90 & 0.280 & 0.88 & 62.26 & 0.0014 & 0.83 \\
\hline$(3.0) \mathrm{Ag} / \mathrm{Cu}-\mathrm{BTC}$ & 83.33 & 0.0093 & 0.99 & 12.87 & 3.96 & 0.79 & 175.84 & 0.177 & 0.81 & 70.39 & 0.0017 & 0.89 \\
\hline (4.0)Ag/Cu-BTC & 90.91 & 0.0156 & 0.99 & 19.47 & 4.76 & 0.80 & 189.29 & 0.535 & 0.83 & 77.64 & 0.007 & 0.91 \\
\hline (5.0)Ag/Cu-BTC & 80.64 & 0.0150 & 0.99 & 18.25 & 4.73 & 0.83 & 197.26 & 0.479 & 0.86 & 73.34 & 0.0007 & 0.89 \\
\hline (4.0)Ag/Zn/Cu-BTC & 101.1 & 0.0245 & 0.99 & 25.94 & 4.92 & 0.54 & 169.26 & 1.117 & 0.61 & 99.07 & 0.0006 & 0.98 \\
\hline (4.0)Ag/Zn/Ni/Cu-BTC & 103.2 & 0.0474 & 0.99 & 32.93 & 5.68 & 0.66 & 186.32 & 3.298 & 0.73 & 99.64 & 0.0001 & 0.98 \\
\hline
\end{tabular}


Table S5. Constants and correlation coefficients of different adsorption models in MIF.

\begin{tabular}{|c|c|c|c|c|c|c|c|c|c|c|c|c|}
\hline \multirow[b]{2}{*}{ Samples } & \multicolumn{3}{|c|}{ Langmuir } & \multicolumn{3}{|c|}{ Freundlich } & \multicolumn{3}{|c|}{ Temkin } & \multicolumn{3}{|c|}{ D-R } \\
\hline & $\begin{array}{c}q_{L} \\
(\mathrm{mg} / \mathrm{g}) \\
\end{array}$ & $\begin{array}{c}K_{L} \\
(\mathrm{~L} / \mathrm{mg}) \\
\end{array}$ & $R^{2}$ & $\begin{array}{c}K_{f} \\
(\mathrm{~L} / \mathrm{g})\end{array}$ & $n$ & $R^{2}$ & $\begin{array}{c}b_{T} \\
(\mathrm{~kJ} / \mathrm{mol})\end{array}$ & $\begin{array}{c}K_{T} \\
(\mathrm{~L} / \mathrm{g}) \\
\end{array}$ & $R^{2}$ & $\begin{array}{c}q_{s} \\
(\mathrm{mg} / \mathrm{g}) \\
\end{array}$ & $\begin{array}{c}K_{D} \\
\left(\mathrm{~mol}^{2} / \mathrm{kJ}^{2}\right)\end{array}$ & $R^{2}$ \\
\hline $\mathrm{Cu}-\mathrm{BTC}$ & 59.52 & 0.0096 & 0.99 & 9.94 & 4.02 & 0.74 & 234.09 & 0.180 & 0.75 & 53.52 & 0.0019 & 0.83 \\
\hline (2.0)Ag/Cu-BTC & 64.31 & 0.0117 & 0.99 & 12.96 & 4.47 & 0.82 & 235.73 & 0.309 & 0.83 & 57.22 & 0.0010 & 0.79 \\
\hline (3.0)Ag/Cu-BTC & 68.21 & 0.0132 & 0.99 & 14.77 & 4.63 & 0.87 & 230.66 & 0.416 & 0.87 & 60.27 & 0.0006 & 0.77 \\
\hline (4.0)Ag/Cu-BTC & 72.94 & 0.0204 & 0.99 & 22.36 & 5.93 & 0.90 & 269.62 & 2.224 & 0.89 & 64.84 & 0.0001 & 0.71 \\
\hline (5.0)Ag/Cu-BTC & 70.97 & 0.0152 & 0.99 & 15.64 & 4.62 & 0.84 & 221.49 & 0.459 & 0.86 & 63.71 & 0.0006 & 0.84 \\
\hline (4.0)Ag/Zn/Cu-BTC & 83.47 & 0.0321 & 0.99 & 25.93 & 5.72 & 0.88 & 235.04 & 3.009 & 0.90 & 74.87 & 0.0004 & 0.80 \\
\hline (4.0)Ag/Zn/Ni/Cu-BTC & 95.41 & 0.0257 & 0.99 & 22.27 & 4.51 & 0.80 & 163.37 & 0.684 & 0.84 & 87.70 & 0.0002 & 0.92 \\
\hline
\end{tabular}

Table S6. Kinetic parameters in ALF for DBT adsorption on Cu-BTC and MMOFs.

\begin{tabular}{|c|c|c|c|c|c|c|c|c|c|c|c|c|c|c|}
\hline \multirow[b]{2}{*}{ Samples } & \multicolumn{6}{|c|}{ Pseudo-first-order rate equation } & \multicolumn{5}{|c|}{ Pseudo-second-order rate equation } & \multicolumn{3}{|c|}{ Intra-particle diffusion model } \\
\hline & $\begin{array}{c}q_{e, \exp } \\
(\mathrm{mg} / \mathrm{g})\end{array}$ & $\begin{array}{c}q_{e, c a l} \\
(\mathrm{mg} / \mathrm{g})\end{array}$ & $\begin{array}{c}K_{l} \\
(1 / \mathrm{min})\end{array}$ & $R^{2}$ & $\begin{array}{c}\Delta q \\
(\mathrm{mg} / \mathrm{g})\end{array}$ & $\begin{array}{l}\Delta q \\
(\%)\end{array}$ & $\begin{array}{c}q_{e, c a l} \\
(\mathrm{mg} / \mathrm{g})\end{array}$ & $\begin{array}{c}K_{2} \\
\text { (g/mg.min) }\end{array}$ & $R^{2}$ & $\begin{array}{c}\Delta q \\
(\mathrm{mg} / \mathrm{g})\end{array}$ & $\begin{array}{l}\Delta q \\
(\%)\end{array}$ & $\begin{array}{c}C \\
(\mathrm{mg} / \mathrm{g})\end{array}$ & $\begin{array}{c}K_{3} \\
\left(\mathrm{mg} / \mathrm{g} \cdot \min ^{1 / 2}\right)\end{array}$ & $R^{2}$ \\
\hline $\mathrm{Cu}-\mathrm{BTC}$ & 50 & 27.07 & -0.0819 & 0.69 & 22.93 & 45.85 & 57.97 & 0.0022 & 0.99 & -7.97 & -15.9 & 14.39 & 5.44 & 0.73 \\
\hline (2.0)Ag/Cu-BTC & 55 & 25.62 & -0.1025 & 0.41 & 29.38 & 53.42 & 62.11 & 0.0025 & 0.99 & -7.11 & -12.9 & 20.41 & 5.27 & 0.76 \\
\hline (3.0) Ag/Cu-BTC & 58 & 23.75 & -0.0978 & 0.52 & 34.25 & 59.05 & 64.43 & 0.0028 & 0.99 & -6.43 & -11.1 & 24.08 & 5.20 & 0.74 \\
\hline (4.0)Ag/Cu-BTC & 65 & 20.40 & -0.0923 & 0.36 & 44.60 & 68.61 & 71.48 & 0.0028 & 0.99 & -6.48 & -9.97 & 28.83 & 5.54 & 0.73 \\
\hline (5.0)Ag/Cu-BTC & 61 & 34.42 & -0.1021 & 0.66 & 26.58 & 43.58 & 67.57 & 0.0027 & 0.99 & -6.57 & -10.8 & 26.07 & 5.31 & 0.76 \\
\hline (4.0)Ag/Zn/Cu-BTC & 70 & 28.52 & -0.1034 & 0.60 & 41.48 & 59.25 & 77.22 & 0.0026 & 0.99 & -7.22 & -10.3 & 30.54 & 6.16 & 0.67 \\
\hline (4.0)Ag/Zn/Ni/Cu-BTC & 82 & 55.83 & -0.1174 & 0.73 & 28.17 & 33.53 & 92.59 & 0.0021 & 0.99 & -8.59 & -10.2 & 36.29 & 7.35 & 0.73 \\
\hline
\end{tabular}


Table S7. Kinetic parameters in ARF for DBT adsorption on $\mathrm{Cu}-\mathrm{BTC}$ and MMOFs.

\begin{tabular}{|c|c|c|c|c|c|c|c|c|c|c|c|c|c|c|}
\hline \multirow[b]{2}{*}{ Samples } & \multicolumn{6}{|c|}{ Pseudo-first-order rate equation } & \multicolumn{5}{|c|}{ Pseudo-second-order rate equation } & \multicolumn{3}{|c|}{ Intra-particle diffusion model } \\
\hline & $\begin{array}{c}q_{e, \exp } \\
(\mathrm{mg} / \mathrm{g})\end{array}$ & $\begin{array}{c}q_{e, c a l} \\
(\mathrm{mg} / \mathrm{g})\end{array}$ & $\begin{array}{c}K_{1} \\
(1 / \mathrm{min})\end{array}$ & $R^{2}$ & $\begin{array}{c}\Delta q \\
(\mathrm{mg} / \mathrm{g})\end{array}$ & $\begin{array}{l}\Delta q \\
(\%)\end{array}$ & $\begin{array}{c}q_{e, c a l} \\
(\mathrm{mg} / \mathrm{g})\end{array}$ & $\begin{array}{c}K_{2} \\
(\mathrm{~g} / \mathrm{mg} \cdot \min )\end{array}$ & $R^{2}$ & $\begin{array}{c}\Delta q \\
(\mathrm{mg} / \mathrm{g})\end{array}$ & $\begin{array}{c}\Delta q \\
(\%)\end{array}$ & $\begin{array}{c}C \\
(\mathrm{mg} / \mathrm{g})\end{array}$ & $\begin{array}{c}K_{3} \\
\left(\mathrm{mg} / \mathrm{g} \cdot \min ^{1 / 2}\right)\end{array}$ & $R^{2}$ \\
\hline $\mathrm{Cu}-\mathrm{BTC}$ & 60 & 52.87 & -0.1364 & 0.83 & 7.13 & 11.89 & 70.67 & 0.0018 & 0.99 & -10.67 & -17.8 & 17.48 & 6.68 & 0.67 \\
\hline (2.0)Ag/Cu-BTC & 65 & 18.93 & -0.0996 & 0.44 & 46.07 & 70.87 & 71.43 & 0.0030 & 0.99 & -6.43 & -9.9 & 28.95 & 5.66 & 0.66 \\
\hline (3.0)Ag/Cu-BTC & 72 & 30.84 & -0.0961 & 0.71 & 41.16 & 57.17 & 78.74 & 0.0028 & 0.99 & -6.74 & -9.4 & 33.20 & 6.03 & 0.69 \\
\hline (4.0)Ag/Cu-BTC & 80 & 25.38 & -0.0855 & 0.58 & 54.62 & 68.28 & 85.76 & 0.0032 & 0.99 & -5.76 & -7.2 & 42.23 & 5.82 & 0.71 \\
\hline (5.0)Ag/Cu-BTC & 76 & 26.08 & -0.0929 & 0.63 & 49.92 & 65.68 & 81.83 & 0.0033 & 0.99 & -5.83 & -7.7 & 39.59 & 5.65 & 0.71 \\
\hline (4.0)Ag/Zn/Cu-BTC & 97 & 33.32 & -0.0825 & 0.60 & 63.68 & 65.64 & 103.52 & 0.0027 & 0.99 & -6.72 & -6.7 & 54.29 & 6.42 & 0.76 \\
\hline (4.0)Ag/Zn/Ni/Cu-BTC & 105 & 39.41 & -0.0874 & 0.62 & 65.59 & 62.47 & 112.87 & 0.0024 & 0.99 & -7.87 & -7.5 & 57.20 & 7.32 & 0.76 \\
\hline
\end{tabular}

Table S8. Kinetic parameters in MIF for DBT adsorption on $\mathrm{Cu}-\mathrm{BTC}$ and MMOFs.

\begin{tabular}{|c|c|c|c|c|c|c|c|c|c|c|c|c|c|c|}
\hline \multirow[b]{2}{*}{ Samples } & \multicolumn{6}{|c|}{ Pseudo-first-order rate equation } & \multicolumn{5}{|c|}{ Pseudo-second-order rate equation } & \multicolumn{3}{|c|}{ Intra-particle diffusion model } \\
\hline & $\begin{array}{c}q_{e, \exp } \\
(\mathrm{mg} / \mathrm{g})\end{array}$ & $\begin{array}{c}q_{e, c a l} \\
(\mathrm{mg} / \mathrm{g})\end{array}$ & $\begin{array}{c}K_{1} \\
(1 / \mathrm{min})\end{array}$ & $R^{2}$ & $\begin{array}{c}\Delta q \\
(\mathrm{mg} / \mathrm{g})\end{array}$ & $\begin{array}{c}\Delta q \\
(\%)\end{array}$ & $\begin{array}{c}q_{e, c a l} \\
(\mathrm{mg} / \mathrm{g})\end{array}$ & $\begin{array}{c}K_{2} \\
(\mathrm{~g} / \mathrm{mg} \cdot \min )\end{array}$ & $R^{2}$ & $\begin{array}{c}\Delta q \\
(\mathrm{mg} / \mathrm{g})\end{array}$ & $\begin{array}{c}\Delta q \\
(\%)\end{array}$ & $\begin{array}{c}C \\
(\mathrm{mg} / \mathrm{g})\end{array}$ & $\begin{array}{c}K_{3} \\
\left(\mathrm{mg} / \mathrm{g} \cdot \min ^{1 / 2}\right)\end{array}$ & $R^{2}$ \\
\hline $\mathrm{Cu}-\mathrm{BTC}$ & 55 & 19.55 & -0.1194 & 0.38 & 35.45 & 64.46 & 63.37 & 0.0023 & 0.99 & -8.37 & -15.2 & 19.91 & 5.80 & 0.69 \\
\hline (2.0)Ag/Cu-BTC & 60 & 30.58 & -0.1011 & 0.67 & 29.42 & 49.03 & 66.71 & 0.0028 & 0.99 & -6.71 & -11.2 & 24.63 & 5.45 & 0.72 \\
\hline (3.0)Ag/Cu-BTC & 64 & 30.16 & -0.0950 & 0.71 & 33.84 & 52.88 & 70.18 & 0.0030 & 0.99 & -6.18 & -9.65 & 29.06 & 5.37 & 0.73 \\
\hline (4.0)Ag/Cu-BTC & 70 & 23.27 & -0.0988 & 0.54 & 46.73 & 66.75 & 75.24 & 0.0036 & 0.99 & -5.24 & -7.49 & 36.83 & 5.16 & 0.69 \\
\hline (5.0)Ag/Cu-BTC & 67 & 23.38 & -0.0957 & 0.62 & 43.62 & 66.11 & 72.25 & 0.0036 & 0.99 & -5.25 & -7.84 & 34.49 & 5.05 & 0.70 \\
\hline (4.0)Ag/Zn/Cu-BTC1 & 80 & 20.06 & -0.0734 & 0.28 & 59.94 & 74.93 & 85.54 & 0.0031 & 0.99 & -5.54 & -6.9 & 43.50 & 5.47 & 0.75 \\
\hline (4.0)Ag/Zn/Ni/Cu-BTC & 90 & 49.42 & -0.0985 & 0.84 & 47.58 & 52.87 & 100.50 & 0.0029 & 0.99 & -10.50 & -11.6 & 52.35 & 6.40 & 0.72 \\
\hline
\end{tabular}




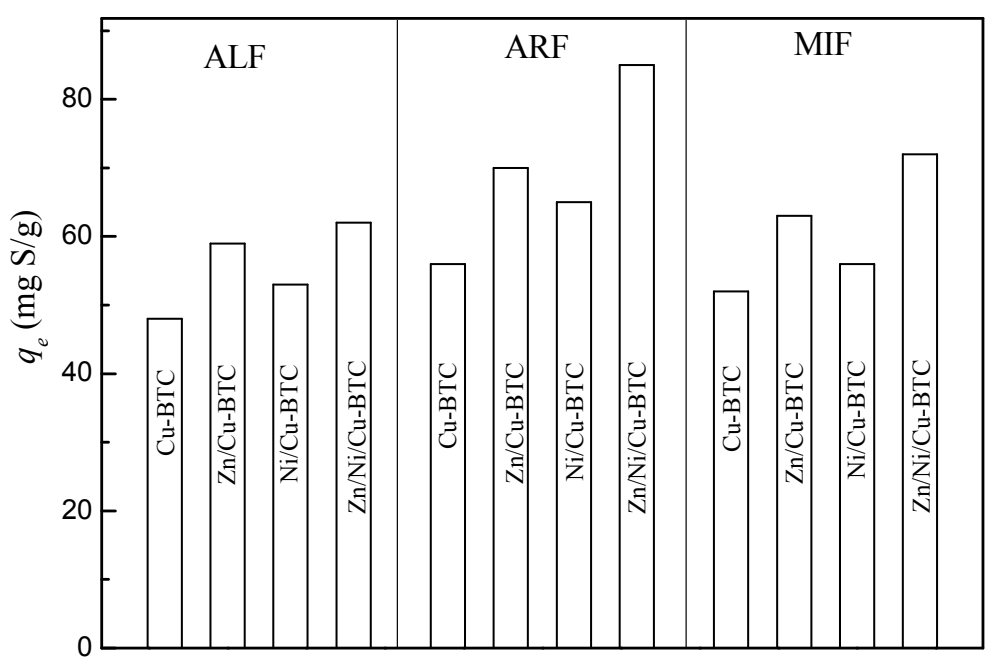

Figure S1. Sulfur uptake capacities of $\mathrm{Cu}-\mathrm{BTC}, \mathrm{Zn} / \mathrm{Cu}-\mathrm{BTC}, \mathrm{Ni} / \mathrm{Cu}-\mathrm{BTC}$ and $\mathrm{Zn} / \mathrm{Ni} / \mathrm{Cu}-\mathrm{BTC}$, in ALF, ARF, and MIF, respectively.

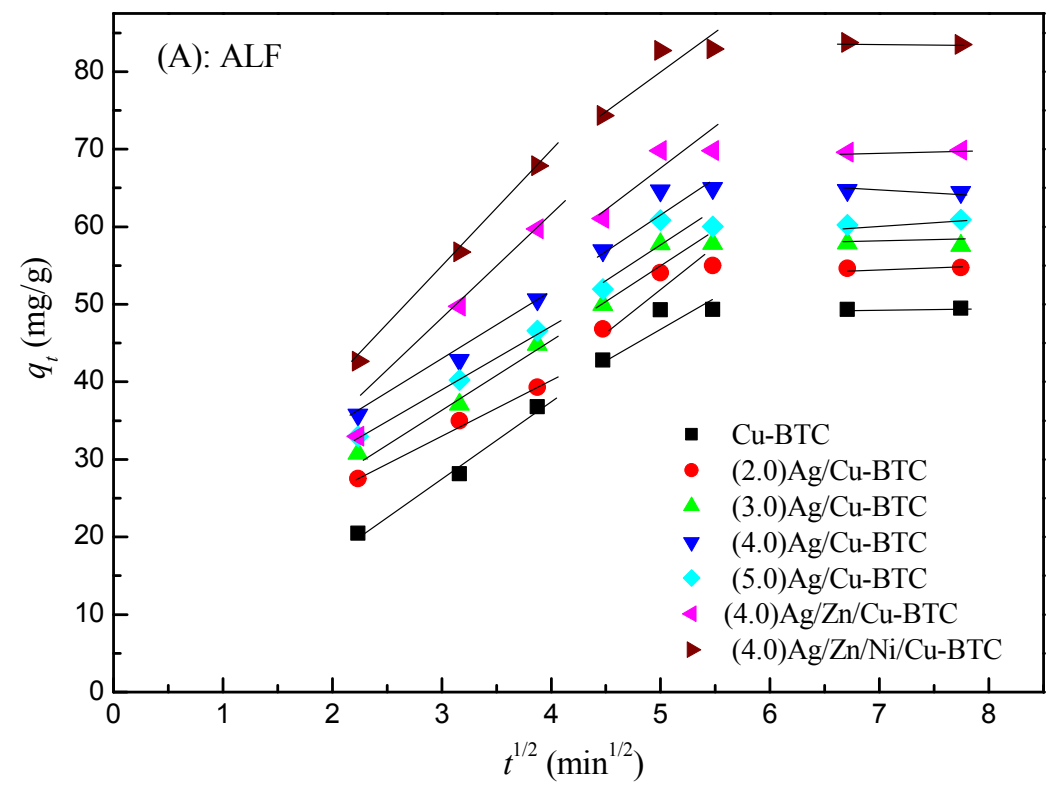



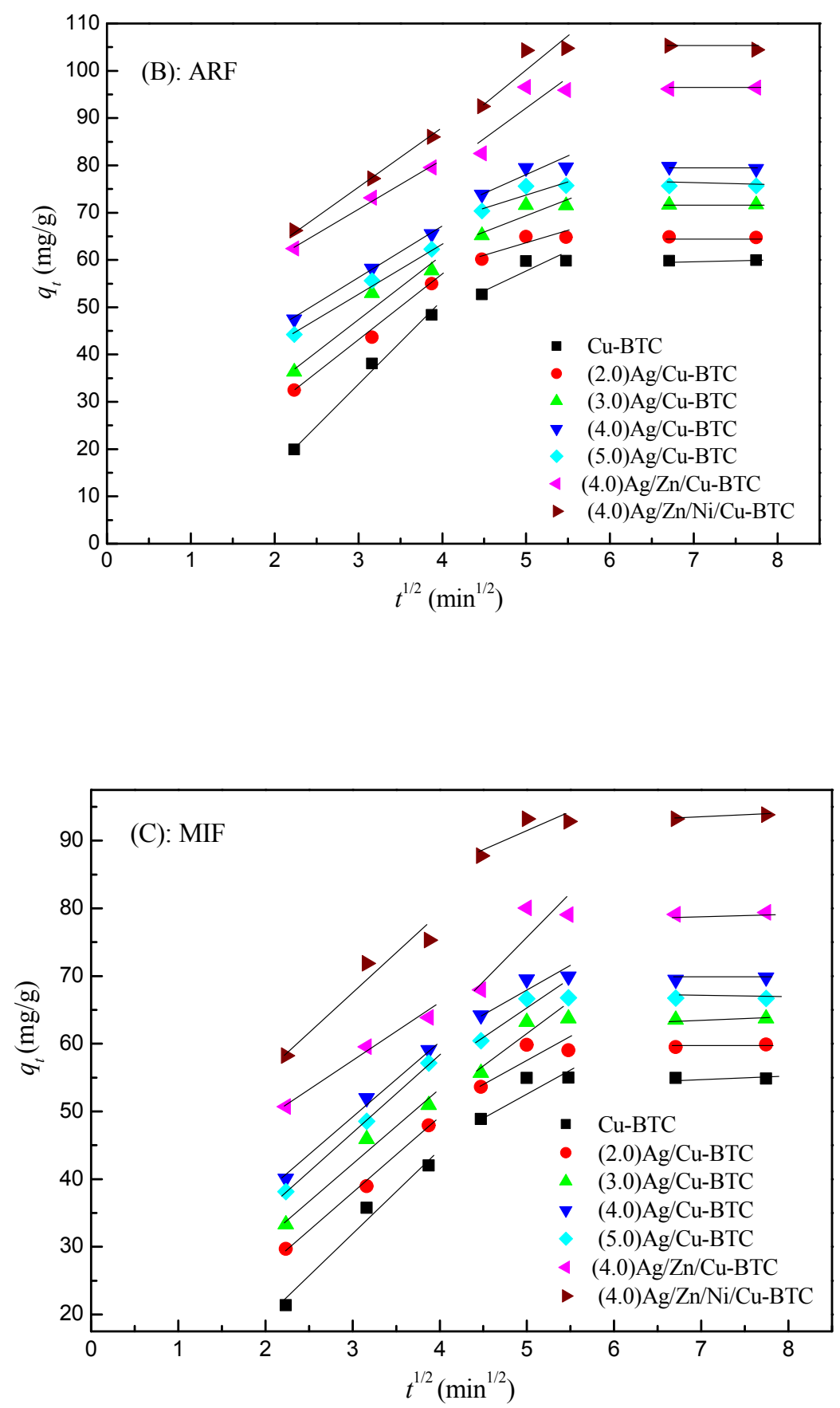

Figure S2. Weber-Morris intra-particle diffusion plots for the adsorption of BT in the ALF, ARF and MIF over MOFs in this work. (A): ALF; (B): ARF; (C): MIF. 


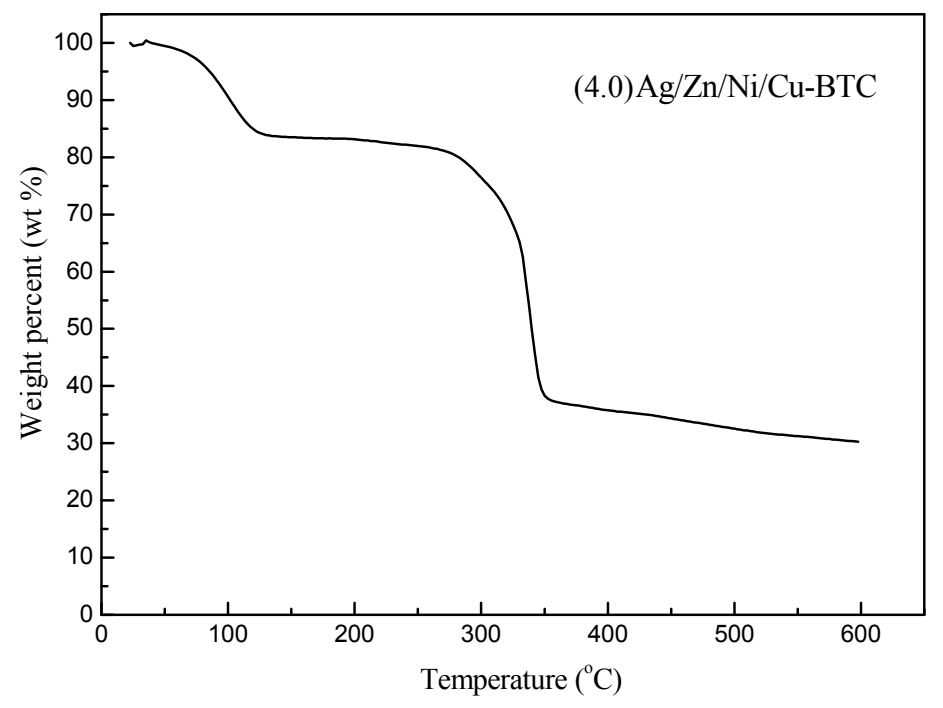

Figure S3. TGA of (4.0)Ag/Zn/Ni/Cu-BTC sample under nitrogen atmosphere.

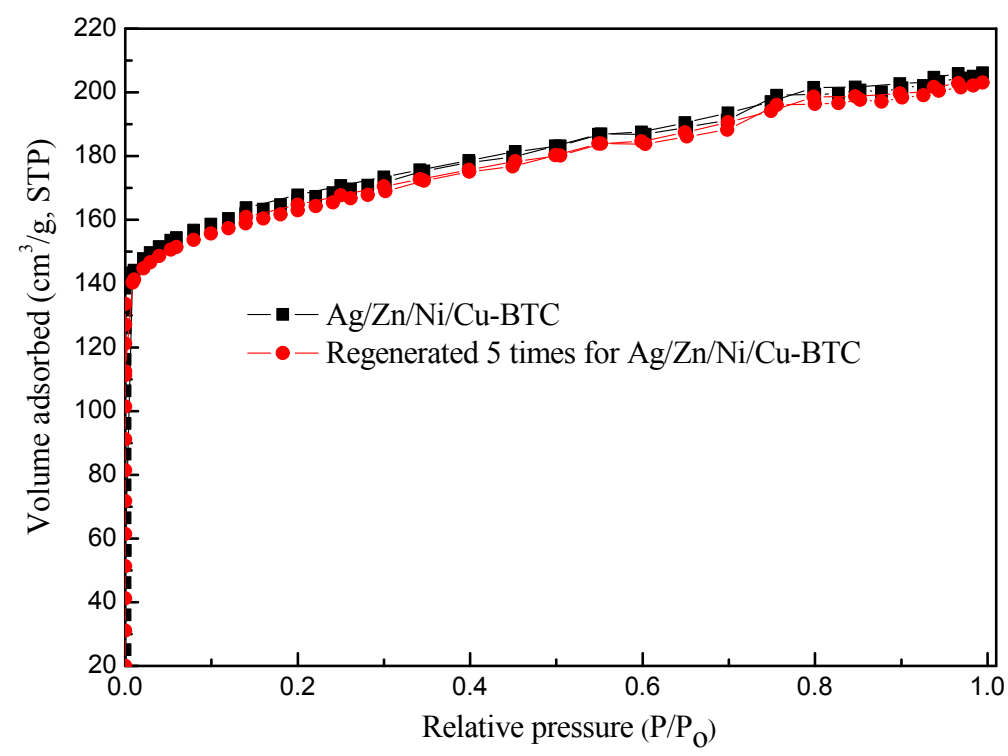

Figure S4. $\mathrm{N}_{2}$ adsorption-desorption curves of (4.0) Ag/Zn/Ni/Cu-BTC before and after regeneration experiments. 


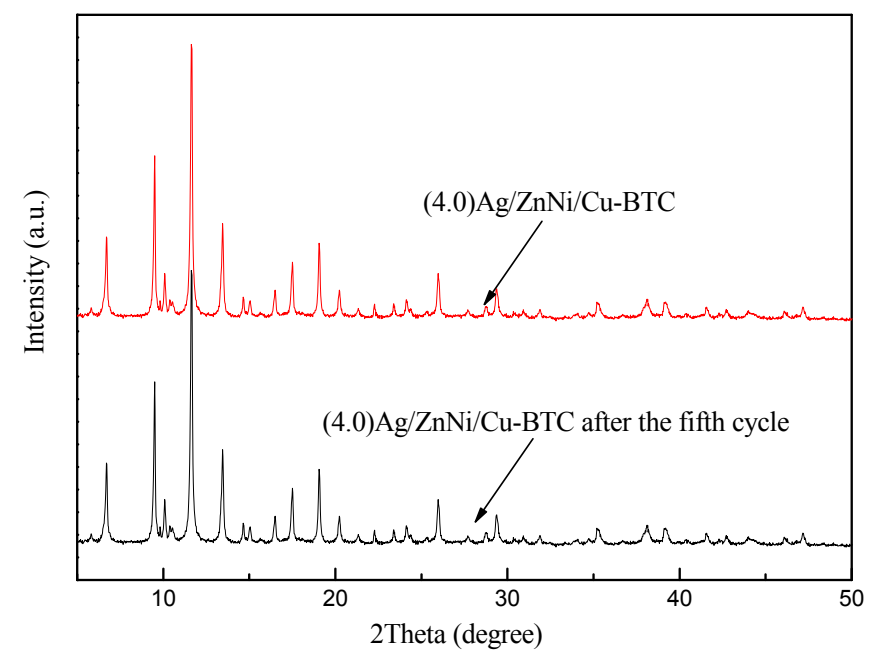

Figure S5. XRD patterns of (4.0) $\mathrm{Ag} / \mathrm{Zn} / \mathrm{Ni} / \mathrm{Cu}-\mathrm{BTC}$ before and after the fifth cycle. 


\section{Related formulas:}

\section{(A) The sulfur uptake capacity was calculated by the formula (1):}

$q_{e}=\frac{W}{M}\left(C_{o}-C_{e}\right)$

where $q_{e}$ is the uptake capacity of sulfur $(\mathrm{mg} / \mathrm{g}), W$ is the mass of model fuel $(\mathrm{g}), M$ is the mass of the sorbent $(\mathrm{g})$, and $C_{o}$ and $C_{e}$ are the initial and final S-concentrations $(\mathrm{mg} / \mathrm{g})$

(B): Adsorption isotherm models used in this study and their linear forms.

\begin{tabular}{cccc}
\hline Isotherm & Nonlinear form & Linear form & Plot \\
\hline Langmuir-I & $q_{e}=\frac{K_{L} C_{e}}{1+K_{L} C_{e}} \quad \frac{C_{e}}{q_{e}}=\frac{1}{q_{L} \cdot K_{L}}+\left(\frac{1}{q_{L}}\right) \cdot C_{e}$ & $\frac{C_{e}}{q_{e}} \operatorname{versus} C_{e}$ \\
Freundlich & $q_{e}=K_{f} C_{e}^{\frac{1}{n}}$ & $\ln q_{e}=\ln K_{f}+\left(\frac{1}{n}\right) \cdot \ln C_{e}$ & $\ln q_{e}$ versus $\ln C_{e}$ \\
Temkin & $e^{q e}=\left(K_{T} C_{e}\right)^{\frac{R T}{b_{T}}}$ & $q_{e}=\frac{R T}{b_{T}} \ln K_{T}+\frac{R T}{b_{T}} \ln C_{e}$ & $q_{e}$ versus $\ln C_{e}$ \\
D-R & $q_{e}=q_{s} e^{\left(-K_{D} \varepsilon^{2}\right)}$ & $\ln q_{e}=\ln q_{s}-K_{D} \varepsilon^{2}$
\end{tabular}

Where $q_{e}$ is the maximum capacity of adsorption in $\mathrm{mg} / \mathrm{g}$; $K_{L}$ is a constant related to the affinity of the binding sites in L/mg;' $K_{f}^{\prime}$ and ' $n$ ' are the measures of adsorption capacity and intensity of adsorption; $R$ is the universal gas constant; $b_{T}$ is related to the heat of adsorption in $\mathrm{kJ} / \mathrm{mol}$. T is the absolute temperature in $\mathrm{K} ; R$ is the universal gas constant; $K_{T}$ is the Temkin constant about the capacity of adsorption in $\mathrm{L} / \mathrm{g} ; q_{s}$ is the D-R isotherm constant in $\mathrm{mg} / \mathrm{g} ; K_{D}$ stands for the constant that is relevant with the adsorption energy in $\mathrm{mol}^{2} / \mathrm{kJ}^{2} ; \varepsilon$ represents the Polanyi potential constant in $\mathrm{kJ} / \mathrm{mol}$;

\section{(C): Adsorption kinetic equations:}

Pseudo-first order model: $\ln \left(q_{e}-q_{t}\right)=\ln \left(q_{e}\right)-K_{1} t$

Pseudo-second order model: $\frac{t}{q_{t}}=\frac{1}{K_{2} q_{e}}+\frac{t}{q_{e}}$

Intra-particle diffusion model: $q_{t}=K_{3} t^{1 / 2}$ 
where $q_{e}$ and $q_{t}(\mathrm{mg} / \mathrm{g})$ are the BT uptake at equilibrium and at time $t$ (min), respectively, $K_{1}(1 / \mathrm{min})$ is the adsorption rate constant, $K_{2}(\mathrm{~g} / \mathrm{mg} \cdot \mathrm{min})$ is the rate constant of second-order equation, $K_{3}\left(\mathrm{mg} / \mathrm{g} \cdot \mathrm{min}^{1 / 2}\right)$ is the intra-particle diffusion rate constant. 